\title{
DreamThrower: Creating, Throwing and Catching Dreams for Collaborative Dream Sharing
}

\author{
Noreen Kamal, Ling Tsou, Abir Al Hajri, and Sidney Fels \\ Department of Electrical and Computer Engineering, University of British Columbia, \\ Vancouver, British Columbia, Canada \\ noreen.k@ubc.ca, tsoul@ece.ubc.ca, \\ alhajria@interchange.ubc.ca, ssfels@ece.ubc.ca
}

\begin{abstract}
The DreamThrower is a novel technology that explores virtually creating, throwing and catching dreams. It detects users' dream state by measuring rapid eye movement (REM). Once the dream state is detected, sound and light stimuli is played to alter the dream. Users report on their dream, and they can send the stimuli that they have used to another person via an on-line website. A working prototype accurately detects REM sleep. Based on preliminary results, the sound and light stimuli were found to have little influence on their dreams. Our prototype's ability to detect REM effectively coupled to a social network to share dream stimuli opens up a fun game environment even if the stimuli itself does not have a significant impact. Instead, user engagement with the social network may be sufficient to alter dreams. Further studies are needed to determine whether stimulus during REM can be created to alter dreams significantly.
\end{abstract}

Keywords: Dream detection, dream creation, dream stimulus, Rapid Eye Movement.

\section{Introduction}

The term Dreamcatcher has become a common place keepsake in North American culture. This idealized and over commercialized object originated from the North American Aboriginal Ojibwa Nation that, traditionally, hung above the bed as a charm to protect children from nightmares, as the dreamcatcher acted as a snare for bad dreams, and only let good dreams through [12]. By taking this analogy of a dreamcatcher and adding the concept of dream throwing and dream creating, we can start to conceive the DreamThrower. Have you ever wondered if you can share your dreams with others? Have you ever considered if by sharing external stimuli, others would have the same or similar dream as you? This research looks at how the knowledge of dream creation and control can be used to create a novel solution to "throw" dreams to other people.

DreamThrower is a fun game concept that alters users' dreams by detecting the dream state and playing external sound and light stimuli, and then coupled with a social network to share these experiences with their friends. Our digital DreamThrower, shown in figure 1 , detects when a person has entered a dream state through eye 
movements using an infrared (IR) sensor. It uses external stimuli such as light and sound to enhance events in one's dreams. When the user is awake, they would diary the events of the dream, as well as rank how pleasant or unpleasant (valence) the dream was. A user would be able to send another person a suggested stimulus via a social network service, or throw their dream to another user.

This rich dream experience combines socialization of dream content and collaboration of sharing dream experiences. This is done by not only using stimuli to alter dream experiences, but also to share these experiences and stimuli with others to better understand if others will have the same or similar dreams. Our prototype's ability to detect rapaid eye movement (REM), which is a phenomenon of extremely active movement of the eye that can be observed while dreaming, effectively coupled to a social network to share dream stimuli opens up the possibility of a fun game environment even if the stimuli itself does not have a significant impact. Instead, user engagement with the social network may be sufficient to alter dreams to discuss with their network of friends.

In the next section, we discuss the related work in the area of dreams and REM, dream stimuli and technologies related to dreams. In section 3, we describe our system design. In section 4, the user evaluation of our prototype is outlined, which is followed by our results (section 6), and finally the future works and conclusion is presented.

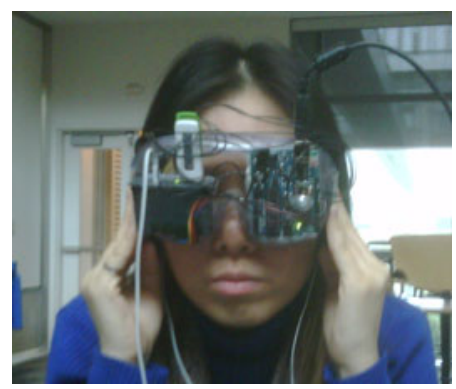

Fig. 1. Photograph of DreamThrower prototype

\section{Related Work}

There has been significant work done in the area of dreaming from the perspective of behavioural psychology and brain science; however, the research done around the design of novel technologies in the domain of dreams is limited. Existing work includes understanding different dream states, lucid dreaming, effects of external stimuli while dreaming, and measuring physiological outputs when dreaming.

There has been research done in understanding different states when dreaming. It is widely accepted that REM strongly correlates to the dreaming state, and that REM dreaming state is qualitatively different than non-REM sleep and waking states [15].

The area of lucid dreaming is a domain that has spurred both academic and nonacademic studies. The concept of lucid dreaming is structured around being aware while one is dreaming, being able to control a dream and being able to remember dreams $[16,18,20]$. Technology has been created to assist a person to be lucid when 
dreaming $[11,18,20,23]$. These technologies include technologies that first detect when a person has fallen asleep such as the NovaDreamer ${ }^{\circledR}$ device $[11,20]$. The DreamSpeaker ${ }^{\circledR}$ provides audible signal and the DreamLight ${ }^{\circledR}$ provides light signals to assist the sleeper to understand that they have fallen into dream state to assist them in maintaining a lucid state while dreaming [11]. Similarly, the Lucid Dream Machine assists people in understanding that they are dreaming to help them in achieving lucid dreams [18]. The REM-Dreamer is another commercial product that works on the same premise but with the added functionality of being able to adjust the intensity of dream stimuli so that it is properly correlated for an individual in a dream state [23]. These technologies use an IR sensor to detect eye movement (REM) to signal when a person is in a dream state; another study looked at methods to detect REM by non-visual means [1]. Our project will use the methods that have been commercialized and studied to detect the dream state by measuring REM and also to understand the concepts of lucid dreaming; although our project will not focus on achieving and maintaining a lucid state when dreaming, it will need to be understood, so that our participants can remember and diary their dream experiences.

Another related research area is in understanding how external stimuli affect dreams. Sound stimuli while sleeping has been studied extensively: verbal sentences during REM sleep can assist in accessing declarative knowledge during sleep and help consolidate knowledge but these verbal cue will not be inserted into dream content [10]; the insertion of verbal content when sleeping also showed that external verbal stimuli are perceived as belonging to the events of the dream [3]; the insertion of verbal stimuli prior to falling asleep was also studied and shown to effect dream content as well as recall after waking [9]; and finally another study revealed that sound stimuli while sleeping cannot be used as an alert [5]. Smell stimuli has also been studied although it has been shown that olfactory senses are very limited while sleeping, and therefore has little effect on dream content $[2,8]$. Finally, the use of light has been used in the lucid dreaming domain to alert a person of a dream state [21]; therefore, we can also appreciate that light or visual stimuli have a cognitive effect while dreaming. Our project will use light and sound including verbal sound as stimuli to change the events of the dream and facilitate dream throwing.

There have also been several studies that look at measuring physiological outputs when dreaming. Various physiological signals correlate to REM or dreaming activity levels $[15,16,22]$. Hobson et al. in 2000 developed the activation-input sourceneuromodulation model (AIM), where electroencephalogram (EEG) activation and firing level of reticular, thalamic and cortical neuron correlates to the activation level; level of presynaptic and postsynaptic inhibition and excitability of sensory motor patterns correlates to internal or external information source; and activity of level of aminergic neurons correlates to mode or organization of data. Holzinger et al. in 2006 studied the electrophysiological differences between lucid and nonlucid dreams in REM sleep by comparing the frequency of EEG signals. EEG signal frequency while sleeping and waking states has been stratified into 7 levels; for example, the delta level is with frequency of 0.1 to $3 \mathrm{~Hz}$, which corresponds to deep sleep and lucid dreaming and the 3-8 Hz is deep relaxation compared to $40 \mathrm{~Hz}$ is high-level information processing [4]. Further studies in understanding EEG signals have studied to further understand the alpha level of EEG activity in REM sleep [6]. Our work will not look at physiological outputs, but rather rely on self-reports of the dream after the person wakes up. 
Another related work that deserves mention is the Dream Communicator, which was one of nine conceptual design proposals for information appliances in [13]. Although the Dream Communicator was only perceived conceptually in this paper and never developed, its concept is to allow distant lovers to enter each other's dream state by stimulating their dreams with sounds or speech; this idea builds on the ability for external stimuli to enter the dream state. Although this design idea presents a portion of the DreamThrower of sending some stimuli to a remote person, it does not provide outputs from the person to visualize their dream experiences, nor the idea of dream crafting and sharing these creations through diary logs.

\section{System Overview}

The DreamThrower is a system that will allow a person to alter their dreams by providing selected light and sound stimuli. The stimuli will only be triggered when REM sleep is detected indicating that the user is dreaming. This unique system will also have an on-line socialization of dreams component, where users can share their dream experiences by self-reporting their dreams, and then "Throw" (or share) these stimuli with their on-line DreamThrower friends.

The system will also include a DreamThrower eye mask component. The eye mask will contain an IR emitter and detector pair to detect REM sleep. Once REM sleep is detected, the selected light and sound stimuli will start until REM sleep ends. Immediately after the dream state ends, the DreamThrower prototype will play a loud sound that says, "wake up", in order to wake the person who is asleep, so that they can report on their dream. Dreams will be self-reported on the DreamThrower website for sharing on-line with friends.

This study does not focus on lucid dreaming or try to invoke lucidity while dreaming. There has been significant work already done in lucid dreaming, so this study will not repeat this body of existing work. The focus of this work will be to invoke dreams by selecting stimuli, and to share dream experiences with others by sharing the same stimuli.

\subsection{Design}

The detailed design for the DreamThrower system is shown in figure 2. The main components of the design are the following:

1. Eye mask: contains the IR emitter and detector, the light stimulus and the sound stimulus

2. Arduino board: provides the power and the processing and control for the dream detection and playing of the stimuli, which is coded in $\mathrm{C}$

3. Computer: loads the stimuli on to the Arduino board and provides the link to the on-line website.

\subsection{REM Detection}

To apply the stimuli at the appropriate time, the DreamThrower system will need to detect when a person is dreaming by detecting the REM state. In order to detect a REM state, an IR light signal will be shined across one eye towards the IR detector. 


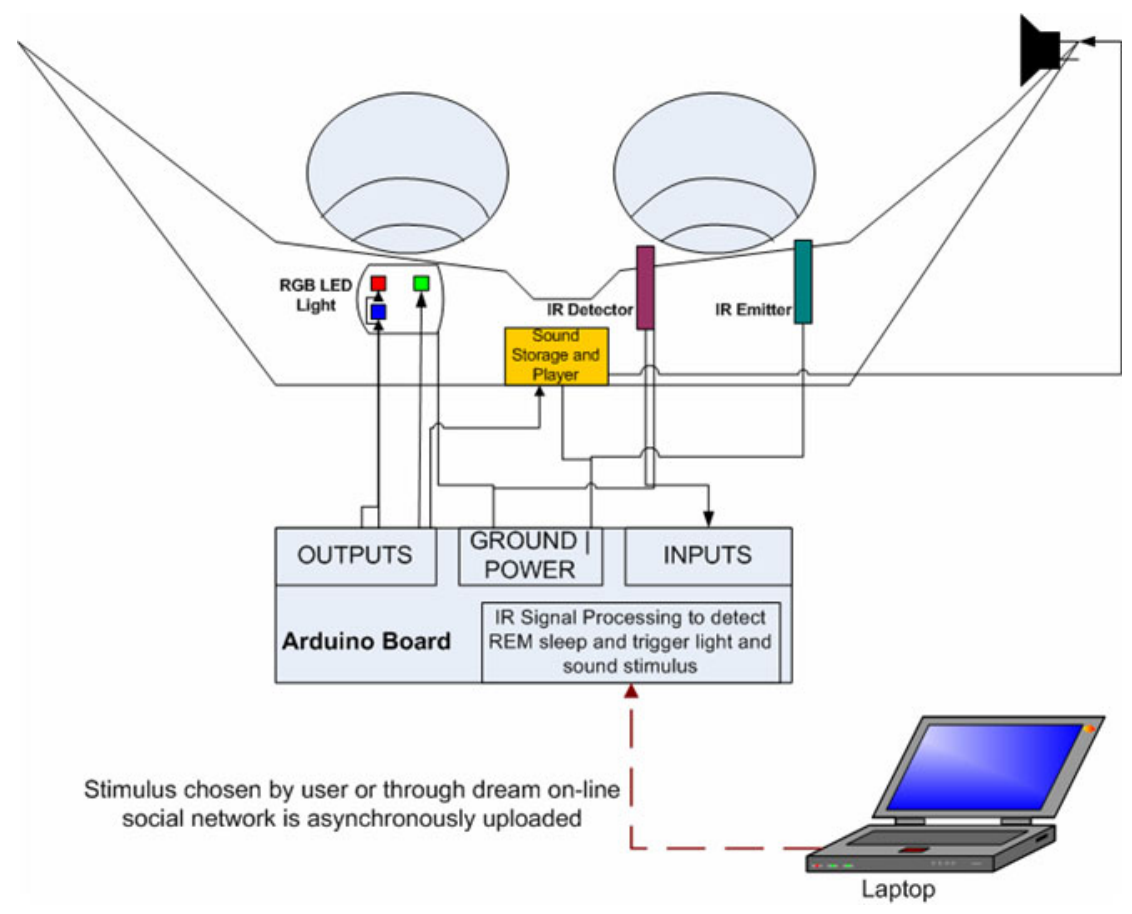

Fig. 2. Detailed design for DreamThrower

When the pupil of the eye is stationary, the IR light will be blocked, and when the pupil moves the IR light will be exposed towards the detector. This is shown in figure 3. The IR detector supplies different levels of voltage to the A/D converter on the Arduino board depends on the amount of IR light received. When the IR light is partially blocked, a low IR signal is detected, and when the IR light is exposed, a high IR signal is detected.

To accurately detect the REM stage, the system performs a series of signal processing algorithms on the sampled IR measurements. A preprocessing step is first done for noise reduction by a combination of windowing and integration. The basic rectangular window is used to divide the signal into blocks of data each contains the IR

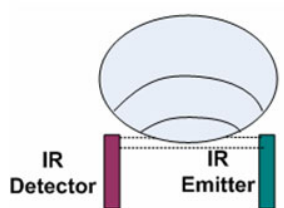

(a)

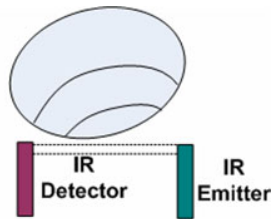

(b)

Fig. 3. REM detection using IR detector and emitter. (a) shows the partial blocking of light when eye is in the position when the eye is not moving, (b) shows the full light signal being received when the eye moves to the side. 
measurements over a small time interval. These blocks are then integrated to provide a single data value for each windowed period. Undesired high frequency noise is filtered, and therefore, makes the signal generated in the REM stage more distinct compared to the base signal where the eyes are quiescent.

The entire REM detection algorithm can be divided into three stages: calibration, REM start detection and REM terminate detection. The calibration stage is used to obtain a baseline for the signal and is triggered after a preset waiting time. It is of import to not start the calibration before the user falls asleep since the eye activities of a person during the REM stage is relatively similar to the activities when he/she is resting but awake [14]. A threshold value, which is required in the later two stages, is set according to this baseline value.

Once the threshold obtained, the system is now ready to perform REM detection. The data points within a window of few seconds are used to observe the level of eye movement activities. After comparing each point with the threshold, a percentage of the number of points excess the threshold level can be calculated. A low percentage indicates that the eye was mostly stationary and pointing straight ahead whereas a high percentage indicates that the eye spent most of the time pointing away from the centre which is caused by extraocular muscle twitches during REM state.

Figure 4 shows the calculated integrated IR signals over one sleep cycle. This plot shows the pre-set waiting time, the calibration step, the non-REM sleep is shown during the low values, and the REM sleep can be seen in the figure as the high values.

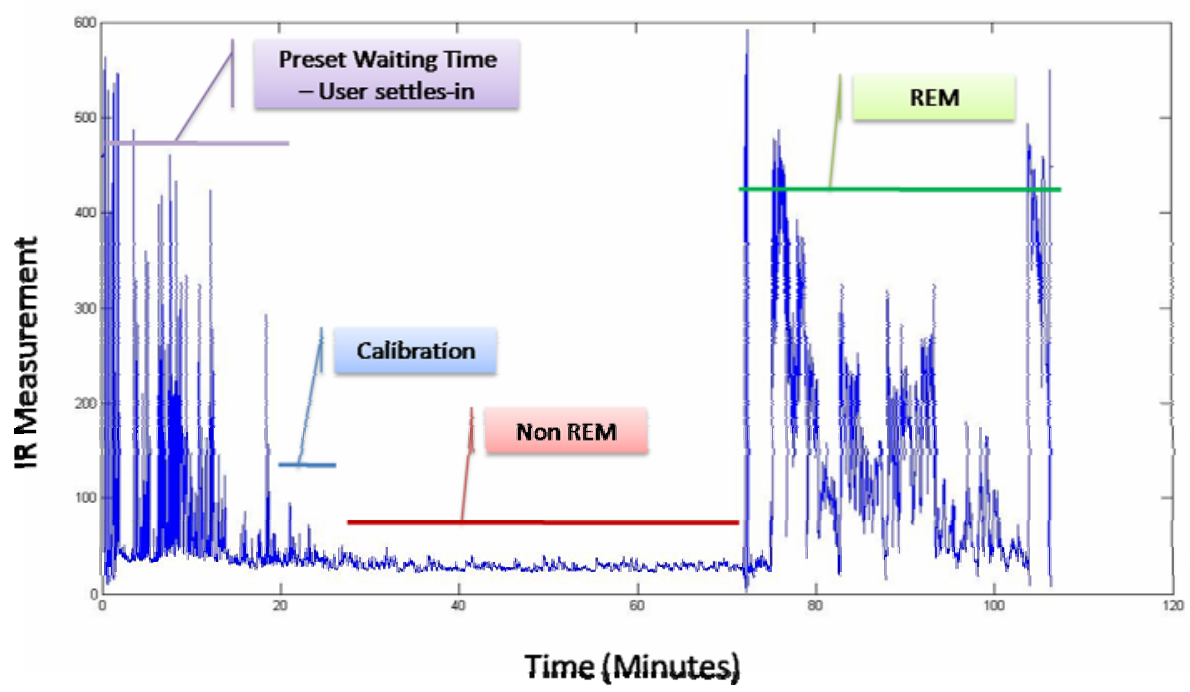

Fig. 4. Plot of integrated IR signal using the DreamThrower prototype. After settling in time (0-20min) we calibrate the background signal $(20-25 \mathrm{~min})$. The subject is sleeping but not dreaming (27-70min) and then enters REM sleep (70-110min). 


\subsection{Sound and Light Stimuli}

Once REM sleep is detected by the DreamThrower, light and sound stimuli that are selected by the user will be played until REM sleep ends. The light stimulus will be sent from the Arduino board to a multi-coloured LED light that will be on the eye mask. This LED light will be able to emit any light colour, which will be flashing on and off at a set frequency. This will be done by controlling the output signals of the Arduino board that are sent to the red, green and blue inputs on the RGB LED light.

The sound stimulus will be triggered when a command is sent from the Arduino board to a VMusic2 module. This VMusic2 module will continuously play the selected sound file from a connected memory stick when REM sleep is detected. The VMusic2 module will also play a sound to wake the user after the REM sleep has ended.

\subsection{Prototype}

A prototype for the DreamThrower was developed. This prototype closely followed the detailed design for the eye mask as described in Section 3 of this paper. Figure 1 shows a photograph of the prototype. Laboratory safety glasses were used for the structure of the DreamThrower eye mask. The IR detector and IR emitter were placed on the left eye of the goggles, and the light stimulus emitter was placed on the right eye of the goggle. The Arduino board, battery, and VMusic2 were placed on-top of the goggles.

The prototype had the following sounds available for the evaluation of the DreamThrower system:

- Jungle sound

- Ocean wave sound

- Heavy rain sound

- Wind sound

It is recognized that these sounds are currently limited to environmental sounds. This will provide focus to our evaluation in understanding the effects of environmental sound stimulus. Further study can be done to include other sounds such as music and verbal speech, as it has been recognized that speech sounds do influence dreams $[9,10]$. Once the system detects that REM sleep has ended, the prototype plays a speech recording, "wake-up" very loudly, so that the user can awaken and report their dream experiences, which was done so that the prototype can be evaluated. It is envisioned that the user would be able to decide if they wish to be awoken after their first dream or second dream or never during a night's sleep. This is important as sleep continuity is of particular concern.

The light stimulus could also be changed in the prototype. The RBG LED light could take any combination of signals from the Arduino program to create a multitude of colours. The current Arduino program will create a flashing light at a set frequency of the colour that is chosen. The sound and light stimuli can be changed directly in the Arduino program and uploaded to the board.

The on-line system was not developed for the prototyping of the system; however, the self-report questions were asked using a questionnaire in the evaluation experiments. 


\section{User Evaluation}

This prototype was used for the user evaluation of the DreamThrower system. The purpose of the evaluation was to answer the following research questions:

1. Does the DreamThrower system accurately detect dreaming state while sleeping and play the stimuli while dreaming?

2. Does the user of DreamThrower feel that the system influenced his/her dreams?

3. Did the user feel that the DreamThrower system could be fun to use and share stimuli?

\subsection{Evaluation}

The system was tested using 3 subjects, who were outside of the research team. The small number of subject for this evaluation was to establish an initial foundational understanding of the DreamThrower system. There were one male and two female subjects.

Each subject participated in 1 experiment. Once again this was to obtain a foundational understanding of the influence of dreams using the stimuli. Table 1 show the type of stimuli that were used on each subject.

Table 1. Stimuli that were used on each subject

\begin{tabular}{llll}
\hline Subject & Gender & Sound & Light \\
\hline Subject 1 & Female & Jungle & Green \\
Subject 2 & Male & Jungle & Green \\
Subject 3 & Female & Ocean & Blue \\
\hline
\end{tabular}

In order to answer the first research question, data was also collected using the Arduino program to help facilitate the evaluation of the system. This included a flag to confirm if the user entered REM sleep and a flag to confirm that the sound and light stimuli was played. This was confirmed by asking the participants if they just had a dream prior to waking-up.

The subjects were informed which sound and light stimuli will be played while they are dreaming. Subjects were informed of the stimuli as this is in line with the usage scenario for DreamThrower, where users download the stimuli onto the device. The eye mask was calibrated for each subject to ensure that the REM detection would function properly for their eye shape. They would wear the mask while sleeping, and when they wake up, each subject was asked to complete an initial questionnaire. This questionnaire inquired if they had a dream and how much of it they could remember.

If the subject did have a dream and remember some of it, they were asked to complete a self-report questionnaire. This questionnaire contained the same inquiries as the on-line self-report page. This will provide an understanding of the dream that the subjects had during the experiment, and it will also provide an analysis of the questions that were chosen for the self-report page. 
Finally, each subject was interviewed to obtain rich data set to qualitatively understand the subjects' thoughts about the DreamThrower system and the amount of influence that it had on their dreams. The purpose of the questionnaires and interview questions were to answer the second and third research questions.

\subsection{Results}

Based on the data retrieved from the Arduino program, we were able to determine that the DreamThrower prototype was able to accurately detect REM sleep and play the selected stimuli for each of the three subjects. This was confirmed by the plot of the IR integrated signal for each user and correlating this with the flag of when the stimuli was played.

The results of each subject's retention and influence of the stimuli on their dream is summarized in table 2 .

Table 2. Results of user evaluation

\begin{tabular}{|c|c|c|c|c|}
\hline Subject & $\begin{array}{l}\text { Remember } \\
\text { Dream? }\end{array}$ & Noticed Stimuli? & $\begin{array}{c}\text { Felt their dream } \\
\text { was influenced } \\
\text { by stimuli? }\end{array}$ & Subject of dream \\
\hline Subject 1 & Little & Yes, sound and light & No & TV Series \\
\hline Subject 2 & Very little & Sound only & No & Sports highlights \\
\hline Subject 3 & Very little & No & No & $\begin{array}{c}\text { DreamThrower } \\
\text { device }\end{array}$ \\
\hline
\end{tabular}

Although the amount of dream content that the subjects remembered varied from little to very little, all three subjects can recall that they had a dream, and they had some recollection of the content of the dream.

The awareness of the stimuli varied for each of the subjects. Subject 1 did notice the light and sound stimuli while dreaming and was awakened by them. The subject 2 only noticed the sound but not the light stimulus, where as subject 3 did not notice any stimuli at all. In the case where the subjects did notice the stimuli, the stimuli were treated by the subjects as actual phenomenon happening in the real world rather than in their dreams. None of the subjects felt that the content of the dream was affected by the stimuli.

No correlation can be observed between the contents of the dreams of the three subjects: subject 3 dreamed about the DreamThrower device itself whereas subject 1 dreamed about a TV series that she watched shortly before starting the experiment, and subject 2 dreamed about sport highlights. All three subjects rated their dreams to be neither disturbing nor pleasant.

The interviews with the subjects also revealed some thoughts on their perception of the level of influence that the device had on their dreams. One subject felt that he/she would like to test the device multiple times: "I think, you have to have a series of experiments in your subjects because it is not easy to get familiar with the device as I was affected by the device itself and not by the stimuli". 
Another subject commented about the comfort of the device itself, which speaks to how the device needs to be improved: "The device itself is too uncomfortable to wear. Also, I was awaken by the stimuli shortly after I started dreaming. '

When the subjects were asked if they found the device and the concept of altering dreams based on stimuli "enjoyable", all three answered "no". Although, two of the three subjects said that it was because the device did not alter their dreams, which suggests that if the device could alter the content of their dreams, they may have found it "enjoyable" to use.

\section{Discussion}

The data that was collected from this preliminary user evaluation confirms that the DreamThrower device does accurately detect the dream state of people while they are sleeping. This is because it accurately detected the REM state of all three of the experiment subjects. Therefore, we can confirm our first research question: the DreamThrower system does accurately detect dreaming state while sleeping and play the stimuli, while dreaming.

However, it is evident that our data set on how sound and light stimuli influence dreams is very limited. Therefore, no hard conclusion can be drawn on whether DreamThrower is able to influence dreams. In order to fully understand how environmental sound influence dreams, we would need to test each subject with each sound and light combination with varying volumes and light intensity as well as light flashing frequency. Additionally, it is evident that each subject had very different interaction with the stimuli when it was play. This suggests the high variability in people to filter out external sounds and light when dreaming. Therefore, a user evaluation on a larger number of subjects is needed to fully understand how the DreamThrower device influences dreams.

It is evident, however, that all three subjects' dreams were not influenced by the device. Some possible reasons why the device did not have an effect on the subjects' dreams:

1. The type of audio/light stimuli chosen was not easily acceptable by humans as the content of their dreams. For example, using a recorded conversation instead of natural sounds may have a greater impact on the subject's dream.

2. For the subjects to interpret the applied stimuli as a part of their dreams might take some practice since humans tends to react (wake up when hearing the alarm clock) or filter out (sleep through the sounds of a thunder storm) external stimulation during sleep.

3. The level of audio volume and light intensity might have an effect. If the volume or light intensity was too high, the subject was likely to be awakening by the stimuli before they have any effect on the dream. However, if the volume or light intensity was too low, the subject would not even have noticed the stimuli.

Furthermore, it is possible that the stimuli did have an effect on the lucidity of the subjects' dreams since all three subjects were able to recall that they in fact had a dream. However, further experiments need to be performed to support this argument. 
It is of importance that one of the subjects had a dream about the DreamThrower device, as it shows that dreams can be influenced by external stimulus. This results shows validity to the idea that dreams can be influenced by stimuli and the concept of DreamThrower shows promise. Additionally, it is also interesting to observe that the content of each subject's dream was based on the individual's activities prior to sleeping and also on their personal interests. Therefore, the stimuli should perhaps be more personalized to the individual rather than being limited in scope.

\section{Future Work and Conclusion}

Based on the results of the preliminary user study, it is evident that the idea of dream sharing and dream influence presents an interesting and entertaining concept. It is clear that more work needs to be done to better understand if sound and light stimuli can influence dream. The use of only environmental sounds, such as ocean and rain, is easy for user to filter out or to understand as external sounds. Therefore, further testing on different sounds such as music and speech sounds may yield different results. This presents an area for future evaluation of the device.

Testing the system on more users, where each user goes through the test several time with different stimuli is needed to better understand which sound and light human subjects would find to influence their dreams the most. Allowing users to develop their own stimuli based on their own interests would also provide an interesting study, as the content of the dreams are personalized to people's individual context, thoughts and activities.

The study focused on the DreamThrower device and only touches on the selfreporting component of the whole system. Therefore, future work should also conceptualize and design the on-line system to diary dreams, collaborate with friend and create/choose sound and light stimuli. Additional studies to understand the placebo effect of providing pre-sleep stimuli through a rich social network.

The extension of entertainment to dreaming by incorporating a rich social network environment to share dream experiences and pre-sleep stimulus presents a novel concept. Additionally, The concept of altering dreams by detecting dream state and providing stimuli is novel and presents a challenging yet interesting concept. By understanding the types of stimuli that will alter the dreams of the majority of people, a new human interface paradigm can emerge. For these reasons, it is worth further study.

\section{References}

1. Agarwal, R., Takeuchi, T., Laroche, S., Gotman, J.: Detection of Rapid-Eye Movements in Sleep Studies. IEEE Transactions on Biomedical Engineering. 52(8), 1390-1396 (2005), doi:10.1109/TBME.2005.851512

2. Badia, P., Wesensten, N., Lammers, W., Culpepper, J., Harsh, J.: Responsiveness to Olfactory Stimuli Presented in Sleep. Physiology \& Behavior 48, 87-90 (1990)

3. Berger, R.J.: Experimental Modification of Dream Content by Meaningful Verbal Stimuli. British Journal of Psychiatry 109, 722-740 (1963)

4. Brain-Wave Machine (1999),

http: / /www. hackcanada.com/homegrown/wetware/brainwave

(Retrieved on January 31, 2010) 
5. Burton, S.A., Harsh, J.A., Badia, P.: Cognitive Activity in Sleep and Responsiveness to External Stimuli. Sleep 11(1), 61-68 (1988)

6. Cantero, J.L., Atienza, M., Salas, R.M.: Spectral Features of EEG Alpha Activity in Human REM Sleep: Two Variants with Different Functional Roles? Sleep 23(6), 1-5 (2000)

7. Carskadon, M.A., Dement, C.W.: Normal Human Sleep: An Overview. In: Kryger, M. (ed.) Principles and practice of sleep medicine, pp. 16-25. W. B. Saunders Co., Philidelphis (1994)

8. Carskadon, M.A., Herz, R.S.: Minimal Olfactory Perception During Sleep: Why Odor Alarms Will Not Work for Humans. Sleep 27(3), 402-405 (2004)

9. Cipolli, C., Fagioli, I., Mazzetti, M., Tuozzi, G.: Incorporation of presleep stimuli into dream content: evidence for consolidation effect on declarative knowledge during REM sleep? Journal of Sleep Research 13, 317-326 (2004)

10. Cipolli, C., Bolzani, R., Tuozzi, G., Fagioli, I.: Active processing of declarative knowledge during REM-sleep dreaming. Journal of Sleep Research 10, 277-284 (2001)

11. Courtney, E.: CEREBREX: DreamWare Specification Document (1997),

http: / /www. cerebrex.com/dream.htm\#dreamspk

(Retrieved on January 29, 2010)

12. Dreamcatcher (2010), http: / / en.wikipedia.org/wiki / Dreamcatcher (Retrieved on January 29, 2010)

13. Gaver, B., Martin, H.: Alternatives: Exploring Information Appliances through Conceptual Design Proposals. In: CHI 2000, April 1 - 6, pp. 209-216 (2000)

14. Herman, J.H., Barker, D.R., Roffwarg, H.P.: Similarity of Eye Movement Characteristics in REM Sleep and the Awake State. Psychophysiology 20(5), 537-543 (1983)

15. Hobson, J.A., Pace-Schott, E.F., Stickgold, R.: Dreaming and the brain: Toward a cognitive neuroscience of conscious states. Behavioral and Brain Science 23(6), 793-842 (2000)

16. Holzinger, B., LaBerge, S., Levitan, L.: Psychophysiological Correlates of Lucid Dreaming. Dreaming 16(2), 88-95 (2006)

17. Kahan, T.L., LaBerge, S.: Lucid dreaming as metacognition: Implications for cognitive science. Consciousness and Cognition: An International Journal 3(2), 246-264 (1994)

18. Ketyung: Lucid dream machine lets you have full control of your dreams (2008), http: / /www. techchee.com/2008/06/20/lucid-dream-machinelets-you-have-full-control-of-your-dream/

(Retrieved on January 29, 2010)

19. LaBerge, S.: Lucid dreaming: Evidence and methodology. Behavioral and Brain Science 23(6), 962-964 (2000)

20. LaBerge, S., Rich, R.S., Wright, D.K., Kottke, D.G.: U.S. Patent Number 5,507,716. Washington, D.C. US Patent and Trade Office (1996)

21. The Lucidity Institute: Lucid Dreaming (2007), http : / www . Iucidity . com (Retrieved on January 31, 2010)

22. Perry, E.K., Piggott, M.A.: Neurotransmitter mechanisms of dreaming: Implication of modulatory system based on dream intensity. Behavior and Brain Science 23(6), 990-1092 (2000)

23. REM-Dreamer - Lucid Dream Induction Device, http: / /remdreamer.com/index.php (Retrieved on January 31, 2010) 